\title{
ON A LINEAR DIFFERENTIAL SYSTEM OF NEUTRAL TYPE
}

\author{
P. CH. TSAMATOS
}

(Received 17 May 1982; revised 27 November 1984)

\begin{abstract}
This paper is concerned with the neutral type differential system with derivating arguments. By decomposing the space of initial functions into classes, it is derived that, for each class, the space of corresponding solutions is of finite dimension. The case of common fixed points of the arguments is also studied.
\end{abstract}

1980 Mathematics subject classification (Amer. Math. Soc.): 34 A 10, 34 A 30, 39 B 99.

\section{Introduction}

In linear ordinary differential systems the solutions can be expressed via the evolution operator, which is a matrix-valued function. For functional differential equations the idea of finding operators which describe the solutions can be found in several references. For more details we mention the book of Hale [3, Chapters 7 and 8]. Recently Karakostas [5] and Staikos and Tsamatos [7] proved that an evolution operator, analogous to the evolution operator of linear differential systems, can be defined for some particular case of solutions of linear differential systems with retarded arguments. This expression of solutions is closely related to the dimension of the space of such solutions.

It is well known that the space of all solutions of a differential system with deviating arguments is infinite dimensional. This constitutes a very significant difference between the ordinary and functional differential systems. This difference creates a basic difficulty in comparing the properties of the solutions of differential systems with deviating arguments with those of ordinary systems. Therefore, a decomposition of the space of solutions of a differential system with

(c) 1986 Australian Mathematical Society $0263-6115 / 86 \$ A 2.00+0.00$ 
deviating arguments into finite dimensional subspaces provides a nice approach to this problem. For a more detailed treatment in that direction, we refer the reader to Norkin [6] and to Staikos and Tsamatos [7].

Here the purpose is to decompose the space of initial functions into classes in such a way that, for each class, the space of corresponding solutions is of finite dimension. From this point of view some results of this paper improve analogous results in [7] and [4]. More precisely, in this paper we consider the differential system

$$
x^{\prime}(t)=\sum_{i=1}^{k} A_{i}(t) x\left[\sigma_{i}(t)\right]+\sum_{i=k+1}^{k+m} A_{i}(t) x^{\prime}\left[\sigma_{i}(t)\right], \quad t \in J,
$$

where $J$ is an open interval of the real line $\mathbb{R}$. The functions $A_{i}(i=1, \ldots, i+m)$ are locally integrable on $J$ and take their values in the space of $n \times n$ matrices over $\mathbb{K}$, where $\mathbf{K}$ stands for the real line $\mathbb{R}$ or for the complex plane $\mathbb{C}$. Also, $\sigma_{i}$ : $J \rightarrow J(i=1, \ldots, k+m)$ are continuous functions.

In what follows, for any $\tau \in J$, the symbol $E(\tau)$ will be used for the smallest interval of the real line which contains $\tau$, and which includes the sets $(-\infty, \tau) \cap$ Rang $\sigma_{i}(i=1, \ldots, k+r)$. Also, the symbol $\operatorname{AC}\left(A, \mathrm{~K}^{n}\right)$ stands for the set of all absolutely continuous $\boldsymbol{K}^{n}$-valued functions with common domain $A \subseteq \mathbb{R}$.

Now, we consider the following initial value problem (I.V.P.)

$$
(L)-(\tau, \varphi) \begin{cases}x^{\prime}(t)=\sum_{i=1}^{k} A_{i}(t) x\left[\sigma_{i}(t)\right]+\sum_{i=k+1}^{k+m} A_{i}(t) x^{\prime}\left[\sigma_{i}(t)\right], \\ \text { for almost all } t \in J \cap[\tau, \infty), \\ x=\varphi \text { on } E(\tau), & \end{cases}
$$

where $\tau \in J$ and $\varphi \in \mathrm{AC}\left(E(\tau), \mathrm{K}^{n}\right)$.

For the existence and uniqueness of solutions to various initial value problems for differential equations of neutral type we refer the reader to [1], [2], [4] and [8].

Definition 1. A $K^{n}$-valued function $x$ defined in $J \cap[\tau, \infty)$ is said to be a solution of the I.V.P. $(L)-(\tau, \varphi)$ if $x$ is absolutely continuous on $J$, and if there exists an extension $\hat{x}$ of $x$ with $\hat{x} \in \operatorname{AC}\left(E(\tau) \cup(J \cap[\tau, \infty))\right.$, $\left.\mathbf{K}^{n}\right)$ such that $x^{\prime}(t)=\sum_{i=1}^{k} A_{i}(t) \hat{x}\left[\sigma_{i}(t)\right]+\sum_{i=k+1}^{k+m} A_{i}(t) \hat{x}^{\prime}\left[\sigma_{i}(t)\right]$ for almost all $t \in J \cap[\tau, \infty)$, and such that $\hat{x}=\hat{\varphi}$ on $E(\tau)$.

2. Evolution operators for the I.V.P. $(L)-(\tau, \varphi)$

Let

$$
\widetilde{\mathrm{AC}}\left(E(\tau), \mathbb{K}^{n}\right)=\operatorname{AC}\left(E(\tau), \boldsymbol{K}^{n}\right)-\left\{\varphi \in \operatorname{AC}\left(E(\tau), K^{n}\right): \varphi(\tau)=0\right\}
$$


We consider now a function $\varphi \in \widetilde{\mathrm{AC}}\left(E(\tau), K^{n}\right)$ such that the coordinates of the vector $\hat{\varphi}(\tau)$ are equal to 1 or 0 .

Definition 2. An $n \times n$-matrix valued function $N=N(\cdot ; \tau, \hat{\varphi})$ defined on $E(\tau) \cup\left(J \cap[\tau, \infty)\right.$ ) (where the entries of $N$ are $\mathbb{K}^{n}$-valued functions) is an evolution operator for the I.V.P. $(L)-(\tau, \hat{\varphi})$ if and only if

(i) $(d / d t) N(t)=\sum_{i=1}^{k} A_{i}(t) N\left[\sigma_{i}(t)\right]+\sum_{i=k+1}^{k+m} A_{i}(d / d t) N\left[\sigma_{i}(t)\right]$ for almost all $t \in J \cap[\tau, \infty)$

(ii) $N=\Phi$ on $E(\tau)$, where $\hat{\Phi}=\operatorname{diag}\left(\hat{\varphi}_{1}, \ldots, \hat{\varphi}_{n}\right)$.

Next, we state Theorems 1 and 2 without proofs since they are similar to those of Theorems 1 and 2 in [8].

THEOREM 1. Let $l>0$ be a positive number. Moreover, we suppose that

(1) there exists a constant $\vartheta, 0 \leqslant \vartheta<1$, such that for all $t \in J$,

$$
\frac{1}{l} \sum_{i=1}^{k}\left\|A_{i}(t)\right\| e^{l\left(\sigma_{i}(t)-t\right)}+\sum_{i=k+1}^{k+m}\left\|A_{i}(t)\right\| e^{l\left(o_{i}(t)-t\right)} \leqslant \vartheta .
$$

Then, if the function $\hat{\varphi} \in \widetilde{\mathrm{AC}}\left(E(\tau), \mathbf{K}^{n}\right)$ has a bounded derivative almost everywhere on $E(\tau)$, there exists exactly one evolution operator $N=N(\cdot ; \tau, \hat{\varphi})$ for the I.V.P. $(L)-(\tau, \hat{\varphi})$ such that

$$
\sup _{t \in J}\left(\|N(t)\| e^{-l(t-\tau)}\right)<\infty .
$$

For the case where the equation $(L)$ is of retarded type, i.e., for every $t \in J$,

$$
\sigma_{i}(t) \leqslant t \quad(i=1, \ldots, k+m)
$$

we have the following two results. The first result is a corollary of Theorem 1.

Corollary 1. Suppose that equation $(L)$ is of retarded type and that $l$ is a positive number. Moreover, we assume that

(2) there exist nonnegative constants $\vartheta_{1}, \vartheta_{2}$ with $\left(\vartheta_{1} / l\right)+\vartheta_{2}<1$, and such that

$$
\sum_{k=1}^{k}\left\|A_{i}(t)\right\| \leqslant \vartheta_{1}, \quad \sum_{i=k+1}^{k+m}\left\|A_{i}(t)\right\|<\vartheta_{2} \quad \text { for all } t \in J .
$$

Then, if the function $\hat{\varphi} \in \widetilde{\mathrm{AC}}\left(E(\tau), K^{n}\right)$ has bounded derivative almost everywhere on $E(\tau)$, there exists exactly one evolution operator for the I.V.P. $(L)-(\tau, \hat{\varphi})$ such that

$$
\sup _{t \in J}\left(\|N(t)\|^{-l(t-\tau)}\right)<\infty
$$


THEOREM 2. Suppose that the equation $(L)$ is of cetarded type and that there exist nonnegative constants $\vartheta_{1}, \vartheta_{2}$ so that

$$
\sum_{i=1}^{k}\left\|A_{i}(t)\right\| \leqslant \vartheta_{1} \quad \text { and } \quad \sum_{i=k+1}^{k+m}\left\|A_{i}(t)\right\| \leqslant \vartheta_{2}<1 \quad \text { for all } t \in J .
$$

Then for the I.V.P. $(L)-(\tau, \hat{\varphi})$ there exists exactly one evolution operator.

An immediate consequence of the existence of the evolution for the above I.V.P. $(L)-(\tau, \hat{\varphi})$ is that the unique solution of this problem (under the conditions of the theorems) can be expressed by the formula

$$
x(t)=N(t) x(\tau)=N(t) \hat{\varphi}(\tau) .
$$

This conclusion is obvious from the definition of the evolution.

THEOREM 3. There exists a partition $\mathscr{C}$ of the space $\widetilde{\mathrm{AC}}\left(E(\tau), \mathbb{K}^{n}\right)$ such that for every class $C \in \mathscr{C}$, there exists an evolution operator $N_{C}$ so that for every $\varphi \in C$ the solution of the I.V.P. $(L)-(\tau, \hat{\varphi})$ can be expressed by the formula

$$
x(t)=N_{C}(t) \varphi(\tau), \quad t \in J \cap[\tau, \infty) .
$$

Proof. We introduce in the space $\widetilde{\mathrm{AC}}\left(E(\tau), \mathbb{K}^{n}\right)$ an equivalence relation by the following definition: $\varphi_{1} \sim \varphi_{2}$ if and only if there exists a nonsingular diagonal $n \times n$-matrix $R$ so that $\varphi_{1}=R \varphi_{2}$.

Clearly, for every $\varphi \in \widetilde{\mathrm{AC}}\left(E(\tau), \mathbf{K}^{n}\right)$, there exists a function $\hat{\varphi} \in \widetilde{\mathrm{AC}}\left(E(\tau), \mathbf{K}^{n}\right)$ so that $\varphi \sim \hat{\varphi}$ and the vector $\hat{\varphi}(\tau) \neq 0$ has coordinates equal to 1 or 0 . More precisely, $\varphi=R \hat{\varphi}$, where $R=\operatorname{diag}\left(\xi_{1}, \ldots, \xi_{n}\right)$, and where

$$
\xi_{i}= \begin{cases}\varphi_{1}(\tau), & \text { if } \varphi_{i}(\tau) \neq 0, \\ 1, & \text { if } \varphi_{i}(\tau)=0\end{cases}
$$

Now, if $N(\cdot ; \tau, \hat{\varphi})$ is an evolution operator for the I.V.P. $(L)-(\tau, \hat{\varphi})$, then the function

$$
x(t)=N(t ; \tau, \hat{\varphi}) R \hat{\varphi}(\tau)=N(t ; \tau, \hat{\varphi}) \varphi(\tau), \quad t \in J \cap[\tau, \infty) .
$$

is the solution of the I.V.P. $(L)-(\tau, \varphi)$. Obviously the evolution operator $N(\cdot ; \tau, \hat{\varphi})$ depends only on the representative $\hat{\varphi}$ of the class $[\hat{\varphi}]$. Thus the above relation for the solution $x$ can be written

$$
x(t)=N_{C}(t) \varphi(\tau) .
$$

where $C=[\hat{\varphi}]$.

REMARK 1. If $N(\cdot ; \tau, \hat{\varphi})=\left(\eta_{i j}(\cdot ; \tau, \hat{\varphi})\right)$ is an evolution operator for the I.V.P. (L) $-(\tau, \hat{\varphi})$, then, since $N$ is continuous and $N(\tau)=\hat{\Phi}(\tau)$, it is clear that $\lim _{t \rightarrow \tau^{+}} \eta_{i j}(\tau)=0$, for $i \neq j$. 
In the following, we denote by $\mathscr{H}_{(\tau, \hat{\varphi})}$ the linear space of the solutions of the initial value problems $(L)-(\tau, C \varphi)$, where $\varphi \in[\hat{\varphi}]$ and where $C$ is a diagonal nonsingular constant $n \times n$-matrix. Because of the above Theorem 3, for every $x \in \mathscr{H}_{(\tau, \hat{\varphi})}$ we have

$$
x(t)=N(t) x(\tau), \quad \tau \in J \cap[\tau, \infty),
$$

where $N$ is the evolution of the I.V.P. $(L)-(\tau, \hat{\varphi})$. Thus we have the following proposition.

Proposition 1. The dimension of the space $\mathscr{H}_{(\tau, \hat{\varphi})}$ is equal to or less than $n$.

Corollary 2. The space $\mathscr{H}_{(\tau, e)}$, where $e(t)=(1, \ldots, 1), t \in E(\tau)$, is of dimension $n$.

REMARK 2. The assumption

$$
\sum_{i=k+1}^{k+m}\left\|A_{i}(t)\right\| \leqslant c_{1}<1 \quad \text { for all } t \in J \cap[\tau, \infty)
$$

is necessary for the uniqueness of the solutions of the I.V.P. $(L)-(\tau, \hat{\varphi})$, with $\varphi \in \mathrm{AC}\left(E(\tau), \mathbb{K}^{n}\right)$. Indeed, the I.V.P.

$$
\begin{aligned}
x^{\prime}(t) & =\frac{-4 t}{t^{2}+4 t-4} x(t / 2)+\frac{3 t^{2}+8 t-4}{t^{2}+4 t-4} x^{\prime}(t / 2), \quad t \in[0,1 / 2], \\
x(0) & =1
\end{aligned}
$$

has the solutions $x_{1}(t)=t+1$ and $x_{2}(t)=t^{2}+1$, and the above assumption fails.

\section{The case of common fixed points of the arguments}

For the equation considered in Remark 2 the point $t=0$ is a common fixed point of the arguments. The case of common fixed points of the arguments is of particular interest, since in this case the continuity of the functions $A_{i}(i=$ $1,2, \ldots, k+m$ ) ensures that the solutions are everywhere differentiable (cf. Hamedani [4]). As Driver [2] has shown, this fact does not occcur in the general case $(L)-(\tau, \cdot)$ if $\tau$ is not a common fixed point of the arguments, even when the $A_{i}(i=1,2, \ldots, k+m)$ are continuous.

In this section we suppose that the functions $A_{i}, i=1,2, \ldots, k+m$, are continuous on $J$, and we set

$$
S=\left\{t \in J: \sigma_{i}(t)=t \text { for all } i=1,2, \ldots, k+m\right\} .
$$


Moreover, we assume that $\sigma_{i}, i=1,2, \ldots, k+m$, satisfy a a "weak monotonicity condition" with respect to the set $S$ of the common fixed points of the arguments, i.e. for any $s \in S$

$$
\sigma_{i}(t) \geqslant s \quad \text { for every } t \geqslant s \quad(i=1,2, \ldots, k+m) .
$$

It is clear that for $\tau \in S$ we have $E(\tau)=\{\tau\}$, and hence the I.V.P. $(L)-(\tau, \varphi)$ considered are of the form $(L)-(\tau, \xi), \xi \in \mathbb{K}^{n}$. By $\mathscr{H}_{\tau}$ we denote the set of all solutions of the initial value problems $(L)-(\tau, \xi)$, where $\xi \in \mathbb{K}^{n}$, and we put

$$
\mathscr{H}_{s}=\bigcup_{\tau \in S} \mathscr{H}_{\tau} \text {. }
$$

A solution $x \in \mathscr{H}_{S}$ is called an $\mathscr{H}_{S}$-solution. The $\mathscr{H}_{S}$-solutions can be expressed by an analogous formula as the solutions of the space $\mathscr{H}_{(\tau, \varphi)}$, where $\tau \in J$ and $\varphi \in \operatorname{AC}\left(E(\tau), \mathbf{K}^{n}\right)$.

Definition 3. An $n \times n$-matrix-valued function $N$ (whose entries are $\mathbb{K}$-valued functions) is an $\mathscr{H}_{s}$ evolution of the system $(L)$ if $\operatorname{Dom} N=\{(t, s) \in J \times S$ : $t \geqslant s\}$ and if, for any $s \in S$,

$$
N(s, s)=I_{n \times n},
$$

(ii)

$$
\begin{aligned}
& \frac{\partial}{\partial t} N(t, s)=\sum_{i=1}^{k} A_{i}(t) N\left(\sigma_{i}(t), s\right)+\sum_{i=k}^{k+m} A_{i}(t) \frac{\partial}{\partial t} N\left(\sigma_{i}(t), s\right) \\
& \quad \text { for every } t \in J \cap[s, \infty) .
\end{aligned}
$$

It is trivial that the existence and uniqueness of the $\mathscr{H}_{s}$-evolution of the system $(L)$ is an immediate consequence of Corollary 1 or Theorem 2. Moreover, any $\mathscr{H}_{S}$-solution of the system $(L)$ can be expressed by the formula

$$
x(t)=N(t, \tau) \xi, \quad t \in J \cap[\tau, \infty),
$$

where $\xi=x(\tau)$.

It is noteworthy that, when $\tau \in S$, the evolution operator $N$ depends on $\tau$ but not on the initial function $\varphi$. More precisely, if $\tau \in S$, then

$$
\bigcup_{\varphi \in \mathrm{AC}\left(E(\tau), \mathbf{K}^{n}\right)} \mathscr{H}_{(\tau, \varphi)}=\mathscr{H}_{(\tau, e)} \text {. }
$$

Thus, by setting $\mathscr{H}_{(\tau, e)}=\mathscr{H}_{\tau}$, we have the following corollary.

COROLlaRY 3. The space $\mathscr{H}_{\tau}$ is of dimension $n$.

Now we give some interesting properties of the $\mathscr{H}_{s}$-evolution. 
Proposition 2. For any pair of fixed points $s_{1}, s_{2}$ in $S$ with $s_{1} \leqslant s_{2}$, and for every $t \in J \cap\left[s_{2}, \infty\right)$, we have

$$
N\left(t, s_{2}\right)=N\left(t, s_{1}\right) N\left(s_{1}, s_{2}\right) .
$$

Proof. This proposition is an immediate consequence of the uniqueness of the solutions of the system $(L)$.

REMARK 2. By induction, we may verify that if $s_{1}, s_{2}, \ldots, s_{v}$ are fixed points in $S$ with $s_{1} \leqslant s_{2} \leqslant \cdots \leqslant s_{p}$, then

$$
N\left(t, s_{1}\right)=N\left(t, s_{\nu}\right) N\left(s_{v}, s_{\nu-1}\right) \cdots N\left(s_{2}, s_{1}\right) \text { for every } t \in J \cap\left[s_{\nu}, \infty\right) .
$$

For any $s \in S$, let $P_{s}$ be the set of the so called "singular points" of the $\mathscr{H}_{S}$-evolution $N$ of $(L)$ with respect to $s$, i.e.

$$
P_{s}=\{\tau \in J \cap[s, \infty): \operatorname{det} N(\tau, s)=0\}
$$

As in [7], we can prove the following proposition.

PROPOSITION 3. If $s_{1}, s_{2}$ in $S$ are such that $s_{1} \leqslant s_{2}$, then $P_{s_{2}} \subseteq P_{s_{1}}$, and, moreover,

$$
\begin{aligned}
& P_{s_{1}} \cap\left[s_{2}, \infty\right)=P_{s_{2}}, \quad \text { if } s_{2} \notin P_{s_{1}}, \\
& J \cap\left[s_{2}, \infty\right) \subseteq P_{s_{1}}, \quad \text { if } s_{2} \in P_{s_{1}} .
\end{aligned}
$$

As an application of the above we give the next corollary, which improves Theorem 1 in [4] for the linear case.

Corollary 4. Let the $n \times n$-matrix-valued functions $A_{i}, i=1,2, \ldots, k+m$, be continuous on $J$, and suppose that for every $t \in J$,

$$
\left\|A_{i}(t)\right\| \leqslant \vartheta_{1} \quad(i=1,2, \ldots, k), \quad \text { and } \quad \sum_{i=k+1}^{k+m}\left\|A_{i}(t)\right\| \leqslant \vartheta_{2},
$$

where the constants $\boldsymbol{\vartheta}_{1}, \vartheta_{2}$ satisfy $\vartheta_{1}>0$ and $0 \leqslant \vartheta_{2}<1$. Moreover, suppose that there exists a point $s \in J$ such that for every $i=1,2, \ldots, k+m$,

(i) $\sigma_{i}(t) \geqslant t$ and $\sigma_{i}(t) \leqslant s$ for all $t \in J \cap(-\infty, s]$,

(ii) $\sigma_{i}(t) \leqslant t$ and $\sigma_{i}(t) \geqslant s$ for all $t \in J \cap[s, \infty)$.

Then, for every $\xi \in \mathbf{K}^{n}$, the I.V.P. $(L)-(s, \xi)$ has a unique solution $x$, with Dom $x=J$, which can be expressed by the formula

$$
x(t)=\hat{N}(t, s) \xi, \quad t \in J,
$$

where $\hat{N}$ is an $n \times n$-matrix-valued function differentiable on $J$. 
Proof. Obviously, the point $s$ is a fixed point of the arguments $\sigma_{i}(i=$ $1,2, \ldots, k+m)$. Moreover, the arguments $\sigma_{i}(i=1,2, \ldots, k+m)$ are of retarded type on $J \cap[s, \infty)$. Therefore, Theorem 1 says that the solution $x$ of the I.V.P. $(L)-(s, \xi)$ can be expressed on $J \cap[s, \infty)$ by the formula

$$
x(t)=N(t, s) \xi, \quad t \in J \cap[s, \infty) .
$$

Now, for the I.V.P. $(L)-(s, \xi)$ on $(-\infty, s] \cap J$, where the arguments $\sigma_{i}(1=$ $1, \ldots, k+m)$ are of advanced type, the transformtion $t \mapsto 2 s-t$ leads to an I.V.P. of retarded type, and so the proof is complete.

REMARK 3. Clearly, the space $\mathscr{H}_{\tau}$ in the above Corollary 4 (i.e., the space of all solutions which are defined at the point $\tau$ and which are global solutions on $J$ ) is of dimension $n$.

\section{References}

[1] S. Czerwik, 'Existence, uniqueness and continuous dependence for the parameter of solutions of a system of differential equations with deviating argument', Ann. Polon. Math. 34 (1977), 269-275.

[2] R. Driver, 'Existence and continuous dependence of solutions of a neutral functional differential equation', Arch. Rational Mech. Anal. 19 (1965), 149-166.

[3] J. Hale, Theory of functional differential equations (Springer-Verlag, New York, 1976).

[4] G. G. Hamedani, 'Global existence of solutions of certain functional-differential equations', Časopis Pěst. Mat. 106 (1981), 48-51.

[5] G. Karakostas, 'Expression of solutions of linear differential equations of retarded type', Boll. Un. Mat. Ital. A 17 (1980), 428-435.

[6] S. B. Norkin, 'Decomposition of the space of solutions of retarded differential equations and the oscillation of solutions', Colloq. Math. Soc. János Bolyai 15, North-Holland, 1977, 335-345.

[7] V. A. Staikos and P. Ch. Tsamatos, 'On linear differential equations with retarded arguments', Math. Nachr. 115 (1984), 167-188.

[8] P. Ch. Tsamatos, 'Existence and uniqueness of solutions of neutral type differential equations', Fasc. Math. 14 (1985), 63-72.

\section{Department of Mathematics}

University of Ioannina

Ioannina

Greece 